Original article

\title{
Leishmania braziliensis isolates differing at the genome level display distinctive features in $\mathrm{BALB} / \mathrm{c}$ mice
}

\author{
Camila Indiani de Oliveira ${ }^{\mathrm{a}, 1}$, Maria Jania Teixeira ${ }^{\mathrm{a}, 1}$, Clarissa Romero Teixeira ${ }^{\mathrm{a}}$, \\ Joílson Ramos de Jesus ${ }^{\mathrm{a}}$, Andréa Bomura Rosato ${ }^{\mathrm{a}}$, João Santa da Silva ${ }^{\mathrm{b}}$, Cláudia Brodskyn ${ }^{\mathrm{a}, \mathrm{c}}$, \\ Manoel Barral-Netto ${ }^{\mathrm{a}, \mathrm{d}}$, Aldina Barral ${ }^{\mathrm{a}, \mathrm{d}, *}$ \\ ${ }^{a}$ Centro de Pesquisas Gonçalo Moniz, Fundação Oswaldo Cruz, Rua Waldemar Falcão, 121, Salvador, BA 40295-001, Brazil \\ ${ }^{\mathrm{b}}$ Faculdade de Medicina de Ribeirão Preto, Universidade de São Paulo, Av. Bandeirantes, 3900, Ribeirão Preto, SP 14049-900, Brazil \\ ${ }^{c}$ Instituto de Ciências da Saúde, Universidade Federal da Bahia, Av. Reitor Miguel Calmon, S/N, Salvador, BA 40110-160, Brazil \\ ${ }^{\mathrm{d}}$ Faculdade de Medicina, Universidade Federal da Bahia, Av. Reitor Miguel Calmon, S/N, Salvador, BA 40110-160, Brazil
}

Received 5 January 2004; accepted 13 May 2004

Available online 23 July 2004

\begin{abstract}
Leishmania braziliensis is the species responsible for the majority of cases of human cutaneous leishmaniasis in Brazil. In the present study, L. braziliensis isolates from two different geographic areas in Brazil were studied by RAPD, using arbitrary primers. We also evaluated other biological features of these two isolates. We compared (a) the clinical features they initiate or not once delivered subcutaneously as stationary-phase promastigotes in the footpad of BALB/c mice; (b) the parasite load in both the footpad and the draining lymph node; (c) the cytokines present in the supernatant of cultures of the cell suspensions from the draining lymph nodes; and (d) the cell types present at the site of parasite delivery. The results show that the L. braziliensis strain from Ceará (H3227) is genotypically different from the L. braziliensis strain from Bahia (BA788). H3227-parasitized mice developed detectable lesions, whereas BA788-parasitized mice did not. Fifteen days post parasite inoculation there was an increase in the numbers of macrophages and lymphocytes in the footpads, whatever the parasite inoculum. Parasite load at the inoculation site—namely the footpad—did not differ significantly; in draining lymph nodes, however, it increased over the period under study. Early after parasite inoculation, the cells recovered from the draining lymph nodes of BA788-parasitized mice produced higher levels of IFN- $\gamma$, a feature coupled to a higher number of NK cells. Later, after the parasite inoculation, there was an increased content of IL-12p70 and IL-10 in the supernatant of cells recovered from the lymph nodes of H3227-parasitized mice. This comparative analysis points out that L. braziliensis isolates differing in their genomic profiles do establish different parasitic processes in BALB/c mice.
\end{abstract}

(C) 2004 Elsevier SAS. All rights reserved.

Keywords: Leishmania braziliensis; Random amplification of polymorphic DNA; Interferon gamma; Interleukin-12p70; Interleukin-10

\section{Introduction}

Leishmania is the etiological agent of leishmaniasis, a parasitic disease with diverse clinical manifestations in human beings and other mammals. The mammalian organism becomes a parasitized host when the sand fly probes the skin for a blood meal, injecting metacyclic promastigotes together with saliva. Leishmania braziliensis parasites usually

\footnotetext{
* Corresponding author. Tel.: +55-71-356-8785x215; fax: +55-71-356-8785x261.

E-mail address: abarral@cpqgm.fiocruz.br (A. Barral).

${ }^{1}$ These authors contributed equally to this work.
}

cause a self-healing ulcer at the site of parasite delivery; however, parasites may also metastasize to the nasopharyngeal tract - a process that is thought to be the starting event leading to mucocutaneous leishmaniasis [1]. More rarely, parasite invasion of the bloodstream results in disseminated skin lesion [2].

Extensive studies using L. major have been interpreted according to the following frame. The resistance or the susceptibility to disease, in different mouse strains, is bound by genetic determinants of the host [3]: resistance is mediated by a Th1-type cellular immune response, characterized by the presence of high levels of IFN- $\gamma$ and low levels of IL-4; susceptibility is mediated by a Th2-type cellular immune 
response, characterized by low levels of IFN- $\gamma$ and high levels of IL-4. Not only do these cytokines act on parasiteloaded cells or further host cells by killing the parasites or preventing their replication, but they also contribute to the inflammatory processes occurring in the parasite-loaded tissue. In contrast to L. major, considerably less experimental work has been conducted with L. braziliensis, probably because most mouse strains do not display lesions at the site of inoculation of L. braziliensis [4,5]. This phenotype (absence of lesion) has been associated with the inability to sustain a strong type 2 immune response [6]. Nonetheless, it has been shown that skin biopsies from patients with cutaneous leishmaniasis caused by L. braziliensis displayed IFN- $\gamma$, TNF- $\alpha$ [7] and iNOS[8]. These cytokines play a key role in the control of the parasite load and, in parallel, contribute to the development of intense inflammatory processes at the parasite inoculation site.

Alternatively, it has been shown that the parasite also plays a role in determining the parasitic process outcome, i.e. disease or long-term asymptomatic parasitism. For example, once located in the skin, the development of L. amazonensis is known to lead to many different clinical presentations, including localized cutaneous lesions, mucocutaneous and visceral leishmaniasis, in humans [9], some of these features being reproduced in mice [10]. More recently, it was shown that genotypic characteristics of L. mexicana could be correlated with features of the clinical disease [11] and that $L$. major isolates from the field show differences in pathogenicity upon inoculation into BALB/c mice [12]. Therefore, variation in the pathogenicity of the parasite, which is related to its genetic diversity, is also an important factor influencing the clinical features of leishmaniasis. Although extensive genetic diversity has been documented in L. braziliensis [13], it was correlated with geographical origin [14,15] and not with clinical manifestations of the disease.

In the present study, we used molecular techniques to examine the genetic polymorphism of L. braziliensis strains isolated from two states in Brazil, namely Ceará and Bahia, located in northeastern Brazil. Both Ceará and Bahia are endemic for cutaneous leishmaniasis caused by L. braziliensis. In Ceará, however, the cutaneous lesion is accompanied and sometimes preceded by an impressive enlargement of regional lymph nodes, whereas in Bahia, such findings have not been documented. We have also examined (a) the cell types present at the site of parasite delivery and (b) the cytokine production after in vitro re-stimulation of the cells recovered from the draining lymph nodes and the cell types present at the site of parasite delivery.

\section{Materials and methods}

\subsection{Mice}

Male 4-6-wk-old BALB/c mice were obtained from CPqGM/FIOCRUZ Animal Facility, where they were main- tained under pathogen-free conditions. The Animal Care and Utilization Committee from CPqGM/FIOCRUZ approved all experimental procedures.

\subsection{Parasite culture}

The L. braziliensis strains MHOM/BR/94/H3227 and MHOM/BR/01/BA788 used were isolated from cutaneous ulcers from patients with cutaneous leishmaniasis, from the states of Ceará and Bahia (northeastern Brazil), respectively, after brief (2-4) passages in culture medium. Both isolates were identified as L. braziliensis by PCR [16] and monoclonal antibodies [17]. Promastigotes were grown in 199 medium (Gibco, Grand Island, NY) at $25^{\circ} \mathrm{C}$ supplemented with $10 \%$ heat-inactivated fetal calf serum, $20 \mathrm{mM}$ Hepes, $4 \mathrm{mM}$ $\mathrm{NaHCO}_{3}, 100 \mathrm{U} / \mathrm{ml}$ of penicillin and $100 \mu \mathrm{g} / \mathrm{ml}$ streptomycin (all from Gibco).

\subsection{The readout assay for typing some signatures of polymorphism of the parasites at the nuclear genomic level}

Genomic DNA extraction, RAPD (Random amplification of polymorphic DNA) was done as described [18]. Briefly, $150 \mathrm{ng}$ of parasite DNA was amplified in a final reaction volume of $25 \mu \mathrm{l}$ containing $10 \mathrm{mM}$ Tris- $\mathrm{HCl} \mathrm{pH} 8.5,1.5 \mathrm{mM}$ $\mathrm{MgCl}_{2}, 50 \mathrm{mM} \mathrm{KCl}, 200 \mu \mathrm{M}$ each dNTP, 1.5 U Taq DNA polymerase (Invitrogen, Carlsbad, CA) and $1.25 \mu \mathrm{M}$ of either primer $3302 \quad\left(5^{\prime}\right.$-CTGATGCTAC-3'); $3303 \quad$ (5'TCACGATGCA-3') or 3304 (5'-GCACTGTCA-3'). The amplification cycles consisted of an initial denaturation step at $95{ }^{\circ} \mathrm{C}$ for $5 \mathrm{~min}$, two cycles with denaturation at $95^{\circ} \mathrm{C}$ for $30 \mathrm{~s}$, annealing at $30^{\circ} \mathrm{C}$ for $2 \mathrm{~min}$ and extension at $72^{\circ} \mathrm{C}$ for 1 min, followed by 33 cycles in which annealing was increased to $40{ }^{\circ} \mathrm{C}$. Ten-microliter samples were analyzed by electrophoresis in $1.5 \%$ agarose gels stained with ethidium bromide.

\subsection{Inoculation of parasites into mice}

Stationary-phase promastigotes were inoculated subcutaneously into the hind left footpad of BALB/c mice at a dose of $10^{6}$ parasites in $20 \mu \mathrm{l}$ of saline. Observation of lesion development was made at weekly intervals and footpad swelling was measured in millimeters by a dial gauge caliper. Lesion size was defined as the increase in footpad thickness after subtraction of the size of the controlateral uninfected footpad.

\subsection{Parasite load estimate}

Parasite load was determined using the quantitative limiting dilution assay as described [19]. Briefly, popliteal lymph nodes draining the infected footpad were aseptically excised and homogenized with a tissue glass grinder in $2 \mathrm{ml}$ of Schneider's medium (Sigma, St. Louis, MO). The homoge- 
nates were serially diluted in Schneider's medium supplemented with $100 \mathrm{U}$ of penicillin/ml, $100 \mu \mathrm{g} / \mathrm{ml}$ of streptomycin $/ \mathrm{ml}, 10 \%$ heat inactivated fetal calf serum (Gibco) and 2\% sterile human urine in 96-well plates containing biphasic blood agar medium. The number of viable parasites was determined from the highest dilution at which promastigotes could be grown out after up to 3 weeks of incubation at $25^{\circ} \mathrm{C}$.

\subsection{Culture of lymph node cells and quantitation}

of cytokine in the culture supernatants

For measurement of in vitro cytokine production, singlecell suspensions of infected footpad draining popliteal lymph nodes were prepared aseptically at 3 and 15 days post infection. The cells were diluted $5 \times 10^{6}$ cells $/ \mathrm{ml}$ and dispensed into 96-well plates with L. braziliensis $\mathrm{H} 3227$ or BA788 live promastigotes (stationary phase), at a ratio of five parasites to one cell, or without parasites, in RPMI-1640 medium (Gibco) containing $2 \mathrm{mM}$ L-glutamine, $100 \mathrm{U}$ of penicillin/ml, $100 \mu \mathrm{g} / \mathrm{ml}$ of streptomycin/ml, $10 \%$ heat inactivated fetal calf serum (Gibco) and $0.05 \mathrm{mM}$ $\beta$-mercaptoethanol. Cell culture supernatants were harvested after $48 \mathrm{~h}$ (for IL-4, IL-10 and IL-12p70 measurement) or $72 \mathrm{~h}$ (for IFN- $\gamma$ measurement) of culture at $37^{\circ} \mathrm{C}$ in $5 \% \mathrm{CO}_{2}$. Levels of IFN- $\gamma$, IL-4, IL-10 and IL-12p70 were determined by ELISA using commercial kits (BD Biosciences, San Diego, CA). The cytokine production of lymph node cells from non-infected animals, upon stimulation with live $L$. braziliensis promastigotes, was below the detection level of the kits used for cytokine detection.

\subsection{Flow cytometric analysis}

To characterize leukocytes present in the inoculation site, infected foot tissue was collected at 3 and 15 days post infection and incubated $1 \mathrm{~h}$ at $37^{\circ} \mathrm{C}$, in RPMI-1640 medium containing $2 \mathrm{mM}$ L-glutamine, $100 \mathrm{U}$ of penicillin $/ \mathrm{ml}$, $100 \mu \mathrm{g} / \mathrm{ml}$ of streptomycin/ml, $10 \%$ heat inactivated fetal calf serum (all from Gibco), 0.28 units/ml of Liberase CI (Roche, São Paulo, SP). Foot tissues were processed in the presence of $0.05 \%$ DNAse (Sigma-Aldrich, St. Louis, MO) using Medimachine (BD Biosciences, San Diego, CA) according to the manufacturer's instructions. After processing, cell viability was assessed by trypan blue exclusion and cells were filtered trough a 50- $\mu \mathrm{m}$ filter and washed before flow cytometry analysis. Viable leukocytes were incubated with antibodies specific to GR-1, Mac-1, NK 1.1, CD3 or MHC class II molecules or with isotype control antibodies (all from BD Biosciences, San Diego, CA) for $30 \mathrm{~min}$ at $4{ }^{\circ} \mathrm{C}$ in the dark. Cells were washed and resuspended in PBS, $1 \%$ formaldehyde. For each sample, 10,000 cells were examined. Data were acquired using a FACScan flow cytometer (BD Biosciences) and analyzed using CellQuest software (BD Biosciences).

\subsection{Statistical analysis}

The data are presented as mean \pm standard error of the mean. The significance of the results was calculated by Student's $t$-test, except for the parasite load analysis, over time, within the BA788- and H337-infected mice groups where ANOVA was used. All analyses were performed using Prism (Graph Pad Software, San Diego, CA) software and a $P$-value $<0.05$ was considered significant.

\section{Results}

\subsection{The BA788 and H3227 L. braziliensis strains display genomic polymorphisms: a study with RAPD}

In preliminary experiments, we investigated the degree of genetic polymorphism by RAPD between six L. braziliensis isolates, two from Ceará (MHOM/BR/94/H3227, MHOM/BR/94/H3456) and four from Bahia (MHOM/ BR/00/BA711, MHOM/BR/02/BA427, MHOM/BR/02/ 806 and MHOM/BR/01/BA788). In these experiments, the genomic profiles obtained with the isolates from Ceará were different from those obtained with the isolates from Bahia (data not shown); data obtained with two representative isolates, namely BA788 (Bahia) and H3227 (Ceará), are shown (Fig. 1). RAPD generated 43 amplified bands, varying from 100-2000 bp in length, of which six are common to the two strains. The most diverse genomic profiles were obtained with primers 3303 and 3304, in which 26 of the 35 amplified fragments were polymorphic. The distinct genomic profiles obtained for strains BA788 and H3227, with the three primers tested here, assess DNA polymorphism between these $L$. braziliensis strains.



3302

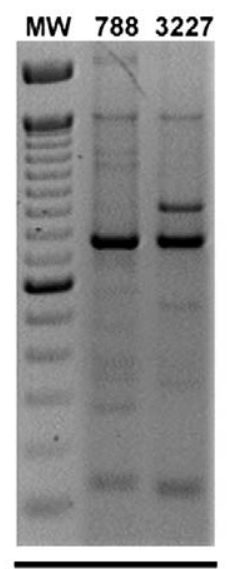

3303

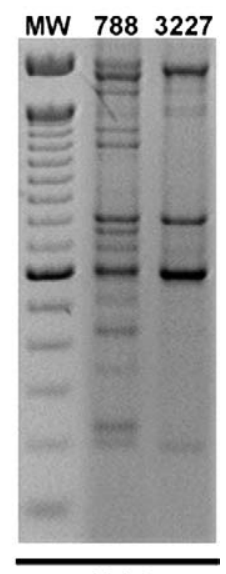

3304
Fig. 1. Genomic profiles of L. braziliensis strains BA788 and H3227. Genomic DNA of each $L$. braziliensis strain was subjected to RAPD using primers 3302, 3303 and 3304. MW; 100 bp ladder DNA size marker. 




Fig. 2. Course of lesion development in BALB/c mice inoculated with $L$. braziliensis strains. BALB/c mice were infected with $10^{6}$ of either $\mathrm{H} 3227$ (black square) or BA788 (white circle) promastigotes in the left hind footpad, and lesion size was measured for 30 days. The footpads of 3-5 mice per group were measured. The data shown, reported as the mean \pm standard error of the mean, are from a single experiment representative of three separate experiments. The asterisk indicates a significant difference between values at the indicated time point as determined by Student's $t$-test $(* P<0.05)$.

\subsection{The amplitude of the transient clinical features} occurring at the site of $\mathrm{L}$. braziliensis delivery differs according to whether the isolate is BA788 or H3227

The genomic polymorphism observed between BA788 and $\mathrm{H} 3227$ prompted us to investigate whether these strains might display differences in pathogenicity upon inoculation into mice of an inbred strain. BALB/c mice received, subcutaneously in the footpad, $10^{6}$ promastigotes of either BA788 or H3227 (Fig. 2). Small increases in footpad thickness were observed early after infection (6 h to 5 days). Mice infected with $\mathrm{H} 3227$ developed lesions detectable at 7 days post inoculation. Lesions progressed steadily, peaked at 15 days post parasite inoculation and gradually resolved 30 days post infection. At this time, we observed the presence of a small nodular lesion associated with cutaneous fibrosis (data not shown). All lesions caused by H3227 L. braziliensis were unulcerated. Even at maximum size, lesions remained nonulcerative and non-necrotic. In mice infected with BA788, in contrast, no similar transient pathogenic processes were noticed. The differences in footpad thickness, between both groups of mice, were significant $(P<0.05)$ in the period ranging from 10-20 days post inoculation.

\subsection{The phenotypic composition of leukocytes present at} the site of BA788 or H3227 L. braziliensis delivery differs according to the isolate

In order to characterize the leukocyte lineages present at the site of parasite delivery, the footpads were excised at 3 and 15 days post parasite inoculation and analyzed by flow cytometry. At 3 days post inoculation, the number of granulocytes, macrophages and lymphocytes is similar in both groups of mice, whereas the number of NK cells is higher in BA788-parasitized mice (Fig. 3). Fifteen days post parasite inoculation, we observed a decrease in the numbers of granu-
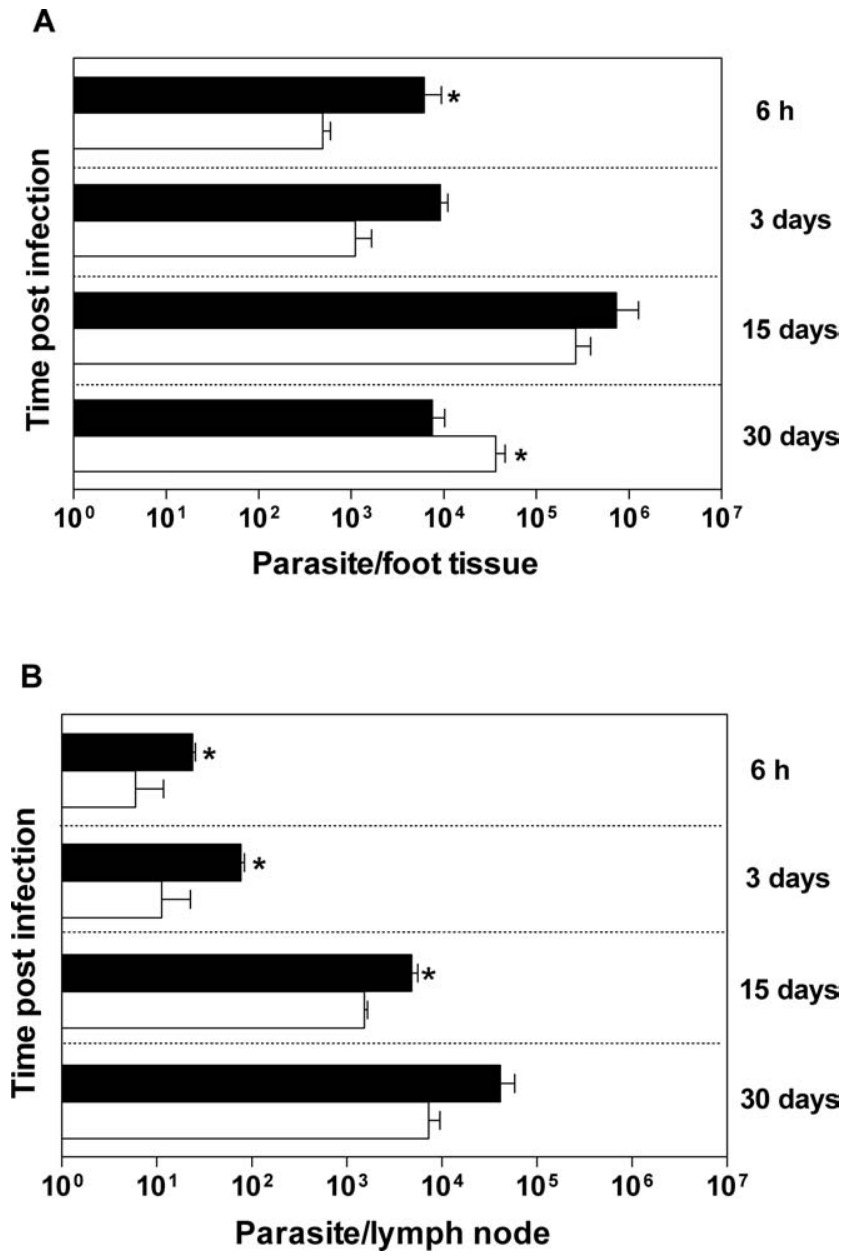

Fig. 3. Phenotypic composition of leukocyte population recovered from the footpad of mice inoculated with $L$. braziliensis strains. BALB/c mice were inoculated with $10^{6}$ promastigotes of either $\mathrm{H} 3227$ (black) or BA788 (white) in the left hind footpad of BALB/c mice. Three (A) and 15 days (B) post inoculation. Leukocytes from inoculated footpads were analyzed by flow cytometry for the presence of lymphocytes, granulocytes, macrophages and NK cells. The data shown are from a single experiment representative of two separate experiments.

locytes and NK cells, in both groups of infected mice. In parallel, there was an increase in the numbers of both macrophages and lymphocytes in the two groups of mice.

\subsection{The BA788 and H3227 L. braziliensis loads differ mainly in the lymph node draining the site where they were delivered}

We also examined if there was a correlation between lesion development and parasite replication. Parasite load was estimated in the inoculated footpads and in the draining lymph nodes, excised at $6 \mathrm{~h}, 3,15$ and 30 days post inoculation, for L. braziliensis BA788 and H3227. In the footpad (Fig. 4A), the parasite load was similar in both groups of mice, but statistically significant differences were observed at $6 \mathrm{~h}$ and 30 days post inoculation. In mice inoculated with H3227 parasites, the parasite load peaked at 15 days, coin- 
A


B
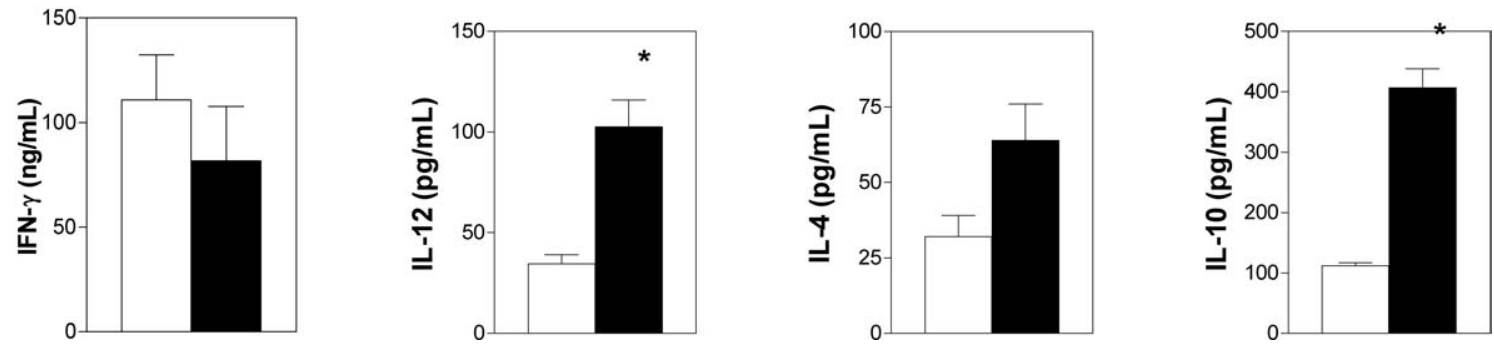

Fig. 4. Parasite load estimate in mice infected with L. braziliensis strains. BALB/c mice were infected with $10^{6}$ of either H3227 (black) or BA788 (white) promastigotes in the left hind footpad. Footpads (A) and popliteal lymph nodes (B) draining the lesion, 3-5 mice per group, were used for analysis of parasite load. The data shown, reported as the mean \pm standard error of the mean, are from a single experiment representative of three separate experiments. The asterisk indicates a significant difference between values from H3227 and BA788 at the same time point as determined by Student's $t$-test $(* P<0.05)$.

ciding with lesion development. However, at this time point, no clinical lesion was observed in mice inoculated with BA788, despite a similar parasite load estimate, indicating dissociation between parasite replication and lesion development. In the draining lymph nodes (Fig. 4B), the parasite load was significantly higher in H3227-parasitized mice, from $6 \mathrm{~h}$ to 15 days post parasite inoculation. Interestingly, in the draining lymph nodes, the parasite load showed a consistent increase in both groups of parasitized mice, indicating that parasites are able to multiply in these secondary lymphoid tissues, regardless of lesion resolution at the upstream site where parasites were inoculated and developed, such as that seen in H3227-parasitized mice.

When comparing the parasite load estimate within the BA788 and H3227 groups, separately, over time, we did not find any significant differences in the footpads inoculated with H3227. In the footpads inoculated with BA788, however, there were statistically significant differences between $6 \mathrm{~h}$ and 15 days $(P<0.01), 3$ and 15 days $(P<0.01)$ and 15 and 30 days post inoculation $(P<0.05)$. Therefore, in the footpad, the parasite load estimate found at 15 days post inoculation is higher than that found at $6 \mathrm{~h}, 3$ days and 30 days post inoculation only in mice receiving the BA788 isolates. In the draining lymph nodes, we did not find any statistically significant differences, between the BA788 and H3227 isolates when comparing the parasite load estimate.

\subsection{At the later time point studied, there is a difference between the ex vivo cytokine profiles displayed by the leukocytes recovered from lymph nodes draining the site of delivery of BA788 or H3227}

The observations that H3227-infected mice controlled the cutaneous lesion and that BA788-infected mice did not develop clinical lesions suggest that, in both cases, a Th1-type cellular immune response had developed. At 3 and 15 days post infection, cells prepared from the lymph nodes draining the infection site were incubated with live BA788 or H3227 promastigotes and were monitored for the production of Th1and Th2-type cytokines. Three days post infection (Fig. 5A), cells from BA788-infected mice produced higher levels of both IFN- $\gamma(P<0.05)$ and IL-12, than cells from H3227infected mice which, on the contrary, produced higher amounts of both IL-4 and IL-10. Fifteen days post-infection (Fig. 5B), Th1-type cytokine production was markedly upregulated in $\mathrm{H} 3227$-infected mice as shown by the significant increase in IL-12 production, in parallel with IFN- $\gamma$. Cells from H3227-infected mice also produced higher amounts of both IL-4 and IL-10, the latter showing a significant upregulation at this time point. This up-regulation in IL-10 production coincides with the time point at which lesion size and parasite load detected in the footpad reached their peak. Moreover, lymph node cell counts were consistently higher in $\mathrm{H} 3227$ L. braziliensis-infected mice than in BA788- 
A

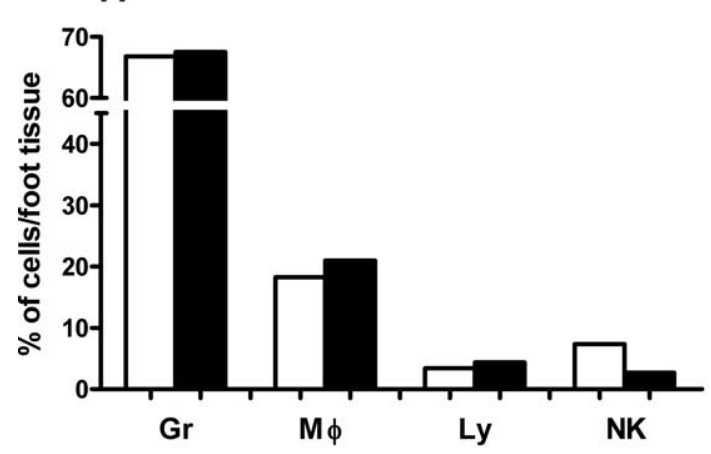

B

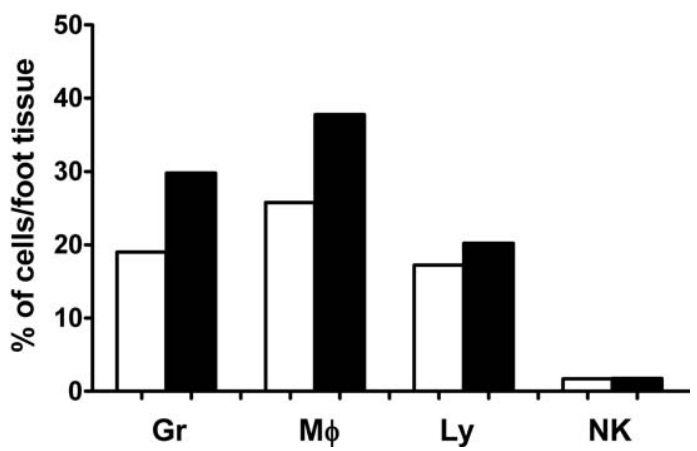

Fig. 5. Cytokine production by mononuclear cells from mice infected with L. braziliensis strains. BALB/c mice were infected with $10^{6}$ promastigotes of either H3227 (black) or BA788 (white) in the left hind footpad. Three (A) and 15 days (B) post infection, mononuclear cells (3-5 mice per group) from popliteal lymph nodes draining the lesion were incubated with live Leishmania promastigotes and the supernatants were assayed for IL-4, IL-10, IL-12p70 and IFN- $\gamma$ production. The data shown, reported as the mean \pm standard error of the mean, are from a single experiment representative of three separate experiments. Cytokine production of lymph node cells from non-infected animals, upon stimulation with live L. braziliensis promastigotes, was below the detection level of the ELISA kits used: IFN- $\gamma$ $(55 \mathrm{pg} / \mathrm{ml}), \mathrm{IL}-4(5.5 \mathrm{pg} / \mathrm{ml}), \mathrm{IL}-10(3 \mathrm{pg} / \mathrm{ml})$ and IL-12p70 (5 pg/ml). The asterisk indicates a significant difference between values as determined by Student's $t$-test $(* P<0.05)$.

infected mice, at both 3 days post infection $(14.8 \times$ $10^{6} \pm 1.6 \times 10^{6}$ vs. $8.1 \times 10^{6} \pm 0.3 \times 10^{6}$, respectively) and at 15 days post infection $\left(24.0 \times 10^{6} \pm 2.8 \times 10^{6}\right.$ vs. $14.7 \times$ $10^{6} \pm 1.3 \times 10^{6}$, respectively).

\section{Discussion}

L. braziliensis parasite populations are extremely diverse, as assessed by RAPD-based readout assays [13]. However, this diversity is less pronounced among parasite populations obtained from areas of geographical proximity [14]. Random amplification of L. braziliensis $\mathrm{H} 3227$ and BA788 DNA generated different genomic profiles, including the presence of amplification fragments not shared between strains. These fragments may constitute nuclear genomic markers for strains from these areas. As far as the BA788 and H3227 L. braziliensis isolates are concerned, the RAPD-based polymorphism can therefore be correlated with their geographical area of sampling, since the $\mathrm{H} 3327$ isolate was sampled from Ceará state, while the BA788 isolate was from Bahia state, both of which are located in northeastern Brazil, where cutaneous leishmaniasis is endemic. In Ceará, the cutaneous lesion is accompanied and sometimes preceded by an impressive enlargement of the regional lymph nodes, and the term bubonic leishmaniasis has been coined to describe this clinical manifestation [20]. Of note, in areas where bubonic leishmaniasis is more often diagnosed, the lymphadenopathy could be the only clinical sign of parasitism [20]. Bubonic leishmaniasis is restricted to L. braziliensis-driven parasitism in Ceará; however, regional lymphadenopathy has also been observed in cutaneous leishmaniasis patients from Bahia $[20,21,22]$. The immunological parameters coupled to these clinical and parasitic features are the presence of high levels of Leishmania-reactive serum antibodies and a leishmanin-positive skin test [21,22].

Relying on the RAPD polymorphism, we considered it relevant to further compare L. braziliensis strains $\mathrm{H} 3227$ and BA788 isolates using laboratory mice as experimental hosts. Transient, non-ulcerated lesions were detected only in H3227-parasitized BALB/c mice. This outcome is characteristic and similar results have been obtained upon the subcutaneous delivery of in vitro grown promastigotes of other $L$. braziliensis isolates to BALB/c mice [23-25]. We did not find any correlation between lesion development and parasite load: indeed the parasite load in the footpad was not significantly different between BA788- and H3227-parasitized mice.

H3227-loaded footpads were processed for histological analysis at 3 and 15 days post inoculation: an inflammatory infiltrate consisting mainly of polymorphonuclear leucocytes and macrophages was noticed. The BA788-loaded footpad sections exhibited a less intense and more transient leukocyte infiltrate (data not shown). Similarly to other L. braziliensis strains, the presence of granulocytes and lymphocytes was noticed during the first week post inoculation; later, many more macrophages and lymphocytes were observed [26].Three days post inoculation, when compared to footpads of H3227-inoculated mice, more NK leukocytes were observed on footpad samples prepared from BA788inoculated mice. NK leucocytes are known to produce IFN- $\gamma$ and may contribute to resistance to L. braziliensis as previously shown for L. major [27]. Moreover, NK cells are detectable as soon as $24 \mathrm{~h}$ post inoculation at the site of $L$. major delivery in resistant mice [28]. Therefore, it is possible that the early and higher IFN- $\gamma$ production observed at the site of BA788 parasite delivery is due to the presence of a higher number of NK cells.

Following the in vitro stimulation of lymph node cells, the ratio of IFN- $\gamma$ to IL-10 was higher when the cells were recovered from BA788-parasitized mice than when the cells were recovered from H3227-parasitized mice, at both 3 days post inoculation (2.28 vs. 0.24 ), respectively, and at 15 days post inoculation ( 0.77 vs. 0.15$)$, respectively. These immunological features are coupled to the outcome of the parasitic 
processes: while BA788-inoculated mice remain lesion-free, the H3227-inoculated mice displayed a transient lesion that heals spontaneously. These features of L. braziliensis parasites in BALB/c mice have been related to their inability to sustain a strong type 2 cytokine profile [6]; this is a main difference with the type 2 cytokine profile driven by L. major when they are delivered in the same conditions as the ones we used in the present study [3].

In conclusion, L. braziliensis isolates differing through their RAPD profiles exhibit differences in clinical features and certain immunological parameters once inoculated into experimental hosts such as BALB/c mice. The differences in clinical features are related to intrinsic properties of the parasite isolates such as ability to induce transient lesion and to recruit different leukocyte populations to the parasiteloaded site. In Ceará state, where H3227 L. braziliensis representative isolate was obtained, the lymphadenopathy, when diagnosed, could precede the lesion form, whereas in Bahia, such lymph node enlargement is not as prominent. Interestingly, this in vivo correlate was reproduced in the present study, since the lymph node cell counts were consistently higher with the H3227 isolate than with the BA788 isolate, at both 3 days and at 15 days post inoculation. These results reinforce the notion that disease manifestation at the site of Leishmania delivery results from a complex interplay between genetically determined parasite as well as host traits. Studies are now in progress to monitor more relevant parasite genetic polymorphism [29].

\section{Acknowledgments}

The authors thank Alexandra R.V. Dias for technical assistance. This study was supported by grants from Conselho Nacional de Desenvolvimento Científico e Tecnológico (CNPq), Coordenação de Aperfeiçoamento de Pessoal de Nível Superior (CAPES) and Fundação de Amparo a Pesquisa do Estado da Bahia (FAPESB). C. Brodskyn, M. Barral-Netto and A. Barral are senior investigators from $\mathrm{CNPq}$ and Instituto de Investigação em Imunologia.

\section{References}

[1] P.D. Marsden, Mucocutaneous leishmaniasis, BMJ 301 (1990) 656 657.

[2] J.M. Costa, P.D. Marsden, E.A. Llanos-Cuentas, E.M. Netto, E.M. Carvalho, A. Barral, A.C. Rosa, C.C. Cuba, A.V. Magalhaes, A.C. Barreto, Disseminated cutaneous leishmaniasis in a field clinic in Bahia, Brazil: a report of eight cases, Am. J. Trop. Med. Hyg 89 (1986) 319-323.

[3] D. Sacks, N. Noben-Trauth, The immunology of susceptibility and resistance to Leishmania major in mice, Nat. Rev. Immunol. 2 (2002) 845-858.

[4] R.A. Neal, C. Hale, A comparative study of susceptibility of inbred and outbred mouse strains compared with hamsters to infection with New World cutaneous leishmaniases, Parasitology 87 (Pt 1) (1983) $7-13$.
[5] G.E. Childs, L.K. Lightner, L. McKinney, M.G. Groves, E.E. Price, L.D. Hendricks, Inbred mice as model hosts for cutaneous leishmaniasis. I. Resistance and susceptibility to infection with Leishmania braziliensis, L. mexicana, and L. aethiopica, Ann. Trop. Med. Parasitol. 78 (1984) 25-34.

[6] G.K. DeKrey, H.C. Lima, R.G. Titus, Analysis of the immune responses of mice to infection with Leishmania braziliensis, Infect. Immun 66 (1998) 827-829.

[7] C. Pirmez, M. Yamamura, K. Uyemura, M. Paes-Oliveira, F. Conceição-Silva, R.L. Modlin, Cytokine patterns in the pathogenesis of human leishmaniasis, J. Clin. Invest 91 (1993) 1390-1395.

[8] C. Bogdan, M. Rollinghoff, A. Diefenbach, The role of nitric oxide in innate immunity, Immunol. Rev. 173 (2000) 17-26.

[9] A. Barral, D. Pedral-Sampaio, G. Grimaldi Junior, H. Momen, D. McMahon-Pratt, A. Ribeiro de Jesus, R. Almeida, R. Badaro, M. Barral-Netto, E.M. Carvalho, W.D. Johnson, Leishmaniasis in Bahia, Brazil: evidence that Leishmania amazonensis produces a wide spectrum of clinical disease, Am. J. Trop. Med. Hyg. 44 (1991) 536-546.

[10] R.P. Almeida, M. Barral-Netto, A.M. De Jesus, L.A. De Freitas, E.M. Carvalho, A. Barral, Biological behavior of Leishmania amazonensis isolated from humans with cutaneous, mucosal, or visceral leishmaniasis in BALB/C mice, Am. J. Trop. Med. Hyg. 54 (1996) 178-184.

[11] M. Berzunza-Cruz, G. Bricaire, S.Z. Romero, R. Perez-Becker, E. Saavedra-Lira, R. Perez-Montfort, M. Crippa-Rossi, O. VelascoCastrejon, I. Becker, Leishmania mexicana mexicana: genetic heterogeneity of mexican isolates revealed by restriction length polymorphism analysis of kinetoplast DNA, Exp. Parasitol. 95 (2000) $277-$ 284.

[12] C. Kebaier, H. Louzir, M. Chenik, A. Ben Salah, K. Dellagi, Heterogeneity of wild Leishmania major isolates in experimental murine pathogenicity and specific immune response, Infect. Immun. 69 (2001) 4906-4915.

[13] E. Cupolillo, H. Momen, G. Grimaldi Jr, Genetic diversity in natural populations of New World Leishmania, Mem. Inst. Oswaldo Cruz 93 (1998) 663-668.

[14] R.F. Gomes, A.M. Macedo, S.D. Pena, M.N. Melo, Leishmania (Viannia) braziliensis: genetic relationships between strains isolated from different areas of Brazil as revealed by DNA fingerprinting and RAPD, Exp. Parasitol 80 (1995) 681-687.

[15] E. Cupolillo, L.R. Brahim, C.B. Toaldo, M.P. de Oliveira-Neto, M.E. de Brito, A. Falqueto, M. de Farias Naiff, G. Grimaldi Jr., Genetic polymorphism and molecular epidemiology of Leishmania (Viannia) braziliensis from different hosts and geographic areas in Brazil, J. Clin. Microbiol. 41 (2003) 3126-3132.

[16] T.M. Castilho, J.J. Shaw, L.M. Floeter-Winter, New PCR assay using glucose-6-phosphate dehydrogenase for identification of Leishmania species, J. Clin. Microbiol. 41 (2003) 540-546.

[17] D. McMahon-Pratt, E. Bennett, J.R. David, Monoclonal antibodies that distinguish subspecies of Leishmania braziliensis, J. Immunol. 129 (1982) 926-927.

[18] A.C. Volpini, V.M. de Azeredo Passos, A.J. Romanha, Attempt to differentiate Leishmania (Leishmania) amazonensis, L. (L.) chagasi, Leishmania (Viannia) braziliensis and L. (V.) guyanensis using the SSR-PCR technique, Parasitol. Res. 87 (2001) 1056-1059.

[19] R.G. Titus, M. Marchand, T. Boon, J.A. Louis, A limiting dilution assay for quantifying Leishmania major in tissues of infected mice, Parasite Immunol 7 (1985) 545-555.

[20] A. de, Q. Sousa, M.E. Parise, M.M. Pompeu, J.M. Coelho Filho, I.A. Vasconcelos, J.W. Lima, E.G. Oliveira, A.W. Vasconcelos, J.R. David, J.H. Maguire, Bubonic leishmaniasis: a common manifestation of Leishmania (Viannia) braziliensis infection in Ceara, Brazil, Am. J. Trop. Med. Hyg 53 (1995) 380-385.

[21] A. Barral, J. Guerreiro, G. Bomfim, D. Correia, M. Barral-Netto, E.M. Carvalho, Lymphadenopathy as the first sign of human cutaneous infection by Leishmania braziliensis, Am. J. Trop. Med. Hyg 53 (1995) 256-259. 
[22] A. Barral, M. Barral-Netto, R. Almeida, A.R. de Jesus, G. Grimaldi Junior, E.M. Netto, I. Santos, O. Bacellar, E.M. Carvalho, Lymphadenopathy associated with Leishmania braziliensis cutaneous infection, Am. J. Trop. Med. Hyg. 47 (1992) 587-592.

[23] J. Samuelson, E. Lerner, R. Tesh, R. Titus, A mouse model of Leishmania braziliensis braziliensis infection produced by coinjection with sand fly saliva, J. Exp. Med 173 (1991) 49-54.

[24] H.C. Lima, R.G. Titus, Effects of sand fly vector saliva on development of cutaneous lesions and the immune response to Leishmania braziliensis in BALB/c mice, Infect. Immun. 64 (1996) 5442-5445.

[25] H.S. Bezerra, M.J. Teixeira, Effect of Lutzomyia whitmani (Diptera: Psychodidae) salivary gland lysates on Leishmania (Viannia) braziliensis infection in BALB/c mice, Mem. Inst. Oswaldo Cruz 96 (2001) 349-351.
[26] K.B. Donnelly, H.C. Lima, R.G. Titus, Histologic characterization of experimental cutaneous leishmaniasis in mice infected with Leishmania braziliensis in the presence or absence of sand fly vector salivary gland lysate, J. Parasitol. 84 (1998) 97-103.

[27] T.M. Scharton, P. Scott, Natural killer cells are a source of interferon- $\gamma$ that drives differentiation of CD4+ T cell subsets and induces early resistance to Leishmania major in mice, J. Exp. Med. 178 (1993) 567-577.

[28] K. Muller, G. van Zandbergen, B. Hansen, H. Laufs, N. Jahnke, W. Solbach, T. Laskay, Chemokines, natural killer cells and granulocytes in the early course of Leishmania major infection in mice, Med. Microbiol. Immunol. 190 (2001) 73-76.

[29] G. Matlashewski, Leishmania infection and virulence, Med. Microbiol. Immunol. (Berl) 190 (2001) 37. 